\title{
From standardization to personalized medicine: Moving beyond cookie-cutter treatment of esophageal cancer
}

\author{
Tamar B. Nobel, MD, a,b and Daniela Molena, $\mathrm{MD}^{\mathrm{a}}$
}

\footnotetext{
From the ${ }^{a}$ Division of Thoracic Surgery, Department of Surgery, Memorial Sloan Kettering Cancer Center, New York, NY; and ${ }^{\mathrm{b}}$ Department of Surgery, Mount Sinai Hospital, New York, NY.

Supported in part by National Institutes of Health/National Cancer Institute Cancer Center Support Grant P30 CA008748.

Disclosures: Authors have nothing to disclose with regard to commercial support

Received for publication June 17, 2018; revisions received June 17, 2018; accepted for publication June 18, 2018. Address for reprints: Daniela Molena, MD, Memorial Sloan Kettering Cancer Center, Thoracic Surgery Service, Department of Surgery, 1275 York Ave, New York, NY 10065 (E-mail: molenad@mskcc.org).

J Thorac Cardiovasc Surg 2018;156:1736-8

$0022-5223 / \$ 36.00$

Copyright $(2018$ by The American Association for Thoracic Surgery

https://doi.org/10.1016/j.jtcvs.2018.06.059
}

Significant advances in diagnosis and therapy for esophageal cancer during the last 4 decades have resulted in improved 5-year overall survival (OS) from $4 \%$ to $22.4 \%{ }^{1}$ In particular, multimodality treatment, including chemotherapy or radiation administered before or after esophagectomy, has been shown to improve survival for patients with locoregional disease relative to surgery alone. ${ }^{2}$ Studies from the 1980s through the 2000s demonstrated the superiority of neoadjuvant chemotherapy ( $\mathrm{nCT}$ ) and chemoradiotherapy (nCRT) relative to surgical resection alone. ${ }^{3}$ As earlier studies were criticized for small sample sizes and poor design, it was the CROSS (ChemoRadiotherapy for Oesophageal cancer followed by Surgery Study) trial published in 2012 that first definitively established the superiority of neoadjuvant therapy combined with surgery relative to surgery alone. ${ }^{4}$ The results of this randomized study of 366 patients with locally advanced esophageal cancer demonstrated a $47 \%$ 5-year OS with the use of nCRT, significantly surpassing previously reported data with the use of $\mathrm{nCT}^{2}$ Subsequently, nCRT became, and has remained, the standard approach for treatment of patients with clinically positive nodal disease and advanced tumor depth $(>\mathrm{T} 2)$ in the United States. Current National Comprehensive Cancer Network guidelines recommend the use of nCRT for all patients with locally advanced esophageal cancer who are good surgical candidates, ${ }^{5}$ and we believe that the adoption of a more standardized approach to this disease has contributed to the observed improved outcome.

As the adoption of nCRT has increased, the use of adjuvant therapy has fallen out of favor for several reasons. Although the results of 2 randomized studies (Medical Research Council Adjuvant Gastric Infusional Chemotherapy [MAGIC], Federation Nationale des Centres de Lutte Contre le Cancer [FNCLCC] and Federation Francophonede Cancerologie Digestive Collaborative [FFCD]) demonstrated pathologic downstaging and up to $13 \%$ improvement in 5-year OS with perioperative colleagues. ${ }^{10}$

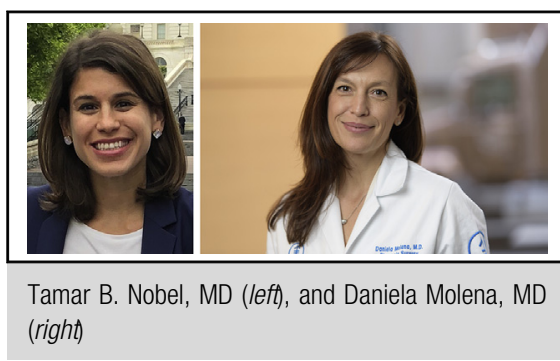

Central Message

Systemic recurrence of esophageal cancer after multimodality treatment remains high. Individualization of care and more aggressive postoperative intervention may improve outcomes over observation.

See Article page 1725 .

chemotherapy (nCT and adjuvant chemotherapy [aCT]) relative to surgery alone, the rates of complete resection and pathologic complete response (pCR) were far lower than those demonstrated after nCRT. ${ }^{6,7}$ Moreover, nearly half of the patients in both studies were unable to receive any postoperative chemotherapy, and many patients were unable to complete the entire recommended dose of aCT because of high reported toxicity. To date, no randomized trials comparing outcomes of neoadjuvant therapy and surgery alone versus addition of postoperative aCT have been completed; however, retrospective studies have suggested that there may be a benefit for patients with persistent nodal disease. ${ }^{8,9}$ This benefit is supported by the article in this issue of the Journal by Samson and

The authors of this study used the National Cancer Data Base (NCDB) to evaluate the role of aCT in patients with residual nodal disease after $\mathrm{nCT}$ or $\mathrm{nCRT}$. In this retrospective study of 9307 patients between 2006 to 2012, a total of 475 of 3100 patients with persistent nodal disease $(15.3 \%)$ received additional adjuvant therapy. Subanalysis demonstrated that patients who received nCRT were less likely to have positive margins than those who received nCT and also had a lower number of positive lymph nodes.

Patients with node-positive disease who received aCT had significantly longer median OS. In a subanalysis of patients with R0 node-positive disease, aCT significantly increased median OS, and similar trends were observed in comparisons across all levels of positive nodal disease (N1-N3). Improved OS in patients who received aCT 
persisted after propensity matching. After controlling for patient and tumor characteristics, a $29 \%$ reduction in mortality was demonstrated in patients who received aCT. Samson and colleagues ${ }^{10}$ conclude that aCT after neoadjuvant therapy may be associated with a survival benefit for patients with positive nodal disease.

The results of this study are encouraging but also demonstrate ongoing difficulties with selection bias with the use of the NCDB. In fact, patients who received aCT were younger, better educated, had higher income, and had better insurance. They were also less likely to have received nCRT. Because the database lacks specificity with respect to chemotherapy regimen and number of cycles administered, it is difficult to determine whether observed residual nodal disease is the result of a more aggressive disease process or an effect of undertreatment. Furthermore, although the data suggest that aCT may be beneficial, difficulty in characterization of postoperative complications with the use of the NCDB challenges the ability to determine the safety of this approach.

Studies evaluating the role of aCT after neoadjuvant therapy for patients with residual nodal disease are limited. For this reason, the National Comprehensive Cancer Network guidelines for patients with persistent nodal disease after nCRT are highly unsatisfying, recommending observation until disease progression in patients with squamous cell carcinoma and consideration of $\mathrm{aCT}$ in patients with adenocarcinoma.

Samson and colleagues ${ }^{10}$ are to be commended for their important contribution to the available knowledge and their attempt to answer 2 important questions that remain partly unanswered: (1) Does aCT after neoadjuvant therapy offer any survival benefit, and if so does the benefit outweigh the risk of treatment? (2) Would all patients benefit from treatment or just a specific subset, and how do we identify that group of patients for which more chemotherapy will result in improved survival? These questions are important today, because although the survival benefits of multimodality treatment have clearly been demonstrated, median OS in patients with locoregional disease has only increased from 6 months in the 1970s to 13 months in the 2000s, despite increased use of neoadjuvant therapy with time. ${ }^{3,11,12}$ Are we offering sufficiently aggressive treatment for such an aggressive disease? There is no question that important prognostic risk factors for survival are directly related to tumor response to therapy and that current therapeutic options for locoregional esophageal cancer are limited in their ability to minimize disease recurrence. The achievement of $\mathrm{pCR}$ is one of the most important determinants of recurrence free and OS after neoadjuvant therapy; however, pCR is achieved in fewer than $30 \%$ of patients. ${ }^{4,13,14}$ Furthermore, recurrence rates remain high even in patients with $\mathrm{pCR} .{ }^{15}$ Once the disease has recurred, prognosis is grim, with reported median survival of 3 to 10 months after diagnosis. ${ }^{16}$ Therein lies a problem-recommendations for watchful waiting in the setting of high recurrence rate and limited treatment options for recurrence juxtaposed with limited options for prevention of recurrence. Most recurrences are systemic, even in patients with $\mathrm{pCR} .{ }^{15}$ Although the benefits of preoperative radiation therapy include reduced dysphagia, increased R0 resection, and pCR, the primary impact of chemotherapy is systemic. This suggests room for improvement with use of additional chemotherapy. The Combination Chemotherapy in Treating Patients With Esophageal Cancer (OE05) trial in fact demonstrated that more aggressive preoperative chemotherapy may result in delayed disease recurrence, but this approach may not be well tolerated in all patient groups. ${ }^{17}$

Furthermore, it has been proposed that the presence of postoperative nodal disease may be more prognostic than residual primary tumor. If persistent positive nodes do represent a more aggressive disease form that has been refractory to neoadjuvant treatment, these patients may not respond to additional chemotherapy. The Metabolic response evalUatioN for Individualisation of neoadjuvant Chemotherapy in oesOphageal and oesophagogastric adeNocarcinoma (MUNICON) trial in fact demonstrated superior survival in patients that had a metabolic response to preoperative chemotherapy relative to those without a response. ${ }^{18}$ Should we completely change our approach and offer more chemotherapy to those with node-negative disease and $\mathrm{pCR}$; that is, those with a response? This concept was explored in a recent NCDB study of aCT after nCRT by Burt and colleagues,${ }^{19}$ who found no difference in $\mathrm{OS}$ in patients treated with aCT after nCRT with pCR. That study did demonstrate similar findings to those of Samson and colleagues ${ }^{10}$ with a $30 \%$ overall reduced risk of death for patients with residual nodal disease who received aCT. It has been shown that higher numbers of positive nodes may predict distant recurrence. ${ }^{20}$ Samson and colleagues ${ }^{10}$ demonstrate that although patients with residual N3 nodal disease had shorter median OS, aCT still improved their prognosis. These studies are likely biased by selection, however, with patients with more extensive disease and lower response to therapy most likely receiving aCT.

Other studies that have used different data sets have shown conflicting results with the use of aCT after neoadjuvant treatment. In one series of 96 patients, aCT after neoadjuvant therapy did not improve survival in patients with node-positive disease. ${ }^{21}$ It should be noted, however, that $89 \%$ of patients received only 1 cycle of chemotherapy. Subsequent studies both demonstrated improved survival and suggested that at least 2 cycles of chemotherapy are needed to achieve a benefit. ${ }^{8,22,23}$ Lack of uniformity in current studies of aCT complicate the ability to draw a meaningful conclusion regarding the associated benefits. 
Esophageal cancer is a heterogeneous disease, and the time has come for individualized patient treatment. Appropriate patient selection for aCT after neoadjuvant treatment remains unknown; however, it is likely not appropriate to offer this treatment to all patients. Current ongoing randomized trials (NEOadjuvant trial in Adenocarcinoma of the oEsophagus and oesophagoGastric junction International Study [Neo-AEGIS], Perioperative Chemotherapy Compared To Neoadjuvant Chemoradiation in Patients With Adenocarcinoma of the Esophagus [ESOPEC], Trial of Preoperative Therapy for Gastric and Esophagogastric Junction Adenocarcinoma [TOPGEAR]) may help to clarify some of these questions, although the answer may lie in tumor genetic characteristics rather than patient and cancer types. ${ }^{24-27}$ In the current setting of poor prognosis for patients with persistent nodal disease and limited therapeutic options, the study by Samson and colleagues ${ }^{10}$ may provide enthusiasm and motivation for us to do more rather than just sit and wait for recurrence.

\section{References}

1. National Cancer Institute. SEER Cancer Statistics Review, 1975-2014. Available at: http://seer.cancer.gov/csr/1975_2014. Accessed June 12, 2018.

2. Cox SJ, O'Cathail SM, Coles B, Crosby T, Mukherjee S. Update on neoadjuvant regimens for patients with operable oesophageal/gastrooesophageal junction adenocarcinomas and squamous cell carcinomas. Curr Oncol Rep. 2017:19:7.

3. Sjoquist KM, Burmeister BH, Smithers BM, Zalcberg JR, Simes RJ, Barbour A, et al; Australasian Gastro-Intestinal Trials Group. Survival after neoadjuvant chemotherapy or chemoradiotherapy for resectable oesophageal carcinoma: an updated meta-analysis. Lancet Oncol. 2011;12:681-92.

4. van Hagen P, Hulshof MC, van Lanschot JJ, Steyerberg EW, van Berge Henegouwen MI, Wijnhoven BPL, et al; CROSS Group. Preoperative chemoradiotherapy for esophageal or junctional cancer. $N$ Engl J Med. 2012; 366:2074-84.

5. National Comprehensive Cancer Network. Esophageal and Esophagogastric Cancers: version 1.2011-May 22, 2018. Available at: https://www.ncen.org/ professionals/physician_gls/pdf/esophageal.pdf. Accessed June 12, 2018.

6. Cunningham D, Allum WH, Stenning SP, Thompson JN, Van de Velde CJ, Nicolson M, et al. MAGIC Trial Participants. Perioperative chemotherapy versus surgery alone for resectable gastroesophageal cancer. $N$ Engl J Med. 2006;355: 11-20.

7. Ychou M, Boige V, Pignon JP, Conroy T, Bouché O, Lebreton G, et al. Perioperative chemotherapy compared with surgery alone for resectable gastroesophageal adenocarcinoma: an FNCLCC and FFCD multicenter phase III trial. J Clin Oncol. 2011;29:1715-21.

8. Glatz T, Bronsert P, Schäfer M, Kulemann B, Marjanovic G, Sick O, et al. Perioperative platin-based chemotherapy for locally advanced esophagogastric adenocarcinoma: postoperative chemotherapy has a substantial impact on outcome. Eur J Surg Oncol. 2015;41:1300-7.

9. Brescia AA, Broderick SR, Crabtree TD, Puri V, Musick JF, Bell JM, et al Adjuvant therapy for positive nodes after induction therapy and resection of esophageal cancer. Ann Thorac Surg. 2016;101:200-8; discussion 208-10.

10. Samson P, Puri V, Lockhart AC, Robinson C, Broderick S, Patterson A, et al. Adjuvant chemotherapy for patients with pathologic node-positive esophageal cancer after induction chemotherapy is associated with improved survival. J Thorac Cardiovasc Surg. 2018;156:1725-35.
11. Njei B, McCarty TR, Birk JW. Trends in esophageal cancer survival in United States adults from 1973 to 2009: a SEER database analysis. J Gastroenterol Hepatol. 2016;31:1141-6.

12. Molena D, Stern M, Blackford AL, Lidor AO. Esophageal cancer treatment is underutilized among elderly patients in the United States. J Gastrointest Surg. 2017;21:126-36.

13. Meredith KL, Weber JM, Turaga KK, Siegel EM, McLoughlin J, Hoffe S, et al. Pathologic response after neoadjuvant therapy is the major determinant of survival in patients with esophageal cancer. Ann Surg Oncol. 2010;17:1159-67.

14. Pasquali S, Yim G, Vohra RS, Mocellin S, Nyanhongo D, Marriott P, et al. Survival after neoadjuvant and adjuvant treatments compared to surgery alone for resectable esophageal carcinoma: a network meta-analysis. Ann Surg. 2017;265:481-91.

15. van Hagen P, Wijnhoven BP, Nafteux P, Moons J, Haustermans K, De Hertogh G, et al. Recurrence pattern in patients with a pathologically complete response after neoadjuvant chemoradiotherapy and surgery for oesophageal cancer. Br J Surg. 2013;100:267-73.

16. Parry K, Visser E, van Rossum PS, Mohammad NH, Ruurda JP, van Hillegersberg R. Prognosis and treatment after diagnosis of recurrent esophageal carcinoma following esophagectomy with curative intent. Ann Surg Oncol. 2015; 22(Suppl 3):S1292-300.

17. Alderson D, Cunningham D, Nankivell M, Blazeby JM, Griffin SM, Crellin A, et al. Neoadjuvant cisplatin and fluorouracil versus epirubicin, cisplatin, and capecitabine followed by resection in patients with oesophageal adenocarcinoma (UK MRC OE05): an open-label, randomised phase 3 trial. Lancet Oncol. 2017; 18:1249-60.

18. Lordick F, Ott K, Krause BJ, Weber WA, Becker K, Stein HJ, et al. PET to assess early metabolic response and to guide treatment of adenocarcinoma of the oesophagogastric junction: the MUNICON phase II trial. Lancet Oncol. 2007; 8:797-805.

19. Burt BM, Groth SS, Sada YH, Farjah F, Cornwell L, Sugarbaker DJ, et al. Utility of adjuvant chemotherapy after neoadjuvant chemoradiation and esophagectomy for esophageal cancer. Ann Surg. 2017;266:297-304.

20. Shaikh T, Zaki MA, Dominello MM, Handorf E, Konski A, Cohen SJ, et al. Patterns and predictors of failure following tri-modality therapy for locally advanced esophageal cancer. Acta Oncol. 2016;55:303-8.

21. Stiles BM, Christos P, Port JL, Lee PC, Paul S, Saunders J, et al. Predictors of survival in patients with persistent nodal metastases after preoperative chemotherapy for esophageal cancer. J Thorac Cardiovasc Surg. 2010;139:387-94.

22. Mirza A, Pritchard S, Welch I. The postoperative component of MAGIC chemotherapy is associated with improved prognosis following surgical resection in gastric and gastrooesophageal junction adenocarcinomas. Int $J$ Surg Oncol. 2013;2013:781742.

23. Luc G, Gronnier C, Lebreton G, Brigand C, Mabrut J, Bail J, et al. Predictive factors of recurrence in patients with pathological complete response after esophagectomy following neoadjuvant chemoradiotherapy for esophageal cancer: a multicenter study. Ann Surg Oncol. 2015;22(Suppl 3):S1357-64.

24. Reynolds J, Preston S, O'Neill B, Baeksgaard L, Griffin SM, Mariette C, et al. ICORG 10-14: NEOadjuvant trial in Adenocarcinoma of the oEsophagus and oesophagoGastric junction International Study (Neo-AEGIS). BMC Cancer. 2017; 17:401.

25. Hoeppner J, Lordick F, Brunner T, Glatz T, Bronsert P, Röthling N, et al. ESOPEC: prospective randomized controlled multicenter phase III trial comparing perioperative chemotherapy (FLOT protocol) to neoadjuvant chemoradiation (CROSS protocol) in patients with adenocarcinoma of the esophagus (NCT02509286). BMC Cancer. 2016;16:503.

26. Leong T, Smithers BM, Michael M, Gebski V, Boussioutas A, Miller D, et al. TOPGEAR: a randomised phase III trial of perioperative ECF chemotherapy versus preoperative chemoradiation plus perioperative ECF chemotherapy for resectable gastric cancer (an international, intergroup trial of the AGITG/TROG/EORTC/NCIC CTG). BMC Cancer. 2015;15:532.

27. Janjigian YY, Sanchez-Vega F, Jonsson P, Chatila WK, Hechtman JF, Ku GY, et al. Genetic predictors of response to systemic therapy in esophagogastric cancer. Cancer Discov. 2018;8:49-58. 